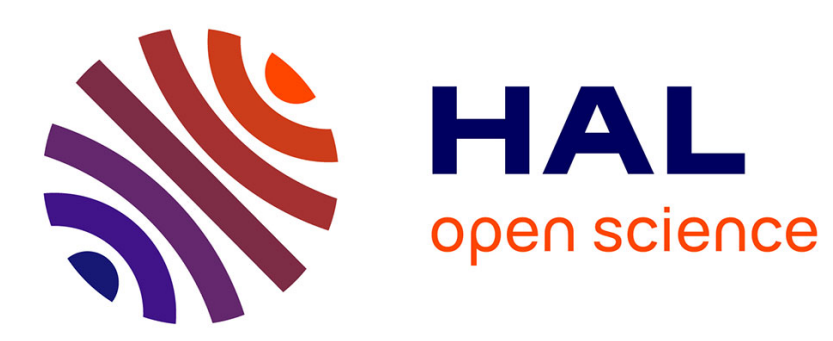

\title{
Perturbations dans la reproduction de la pyrale du maïs, Ostrinia nubilalis Hb. (Lépidoptère Pyralidae), induites par la présence de la phéromone sexuelle de synthèse en conditions de laboratoire
}

\author{
Jacques Pierre Stockel, Jean-Claude Meymerit
}

\section{To cite this version:}

Jacques Pierre Stockel, Jean-Claude Meymerit. Perturbations dans la reproduction de la pyrale du maïs, Ostrinia nubilalis Hb. (Lépidoptère Pyralidae), induites par la présence de la phéromone sexuelle de synthèse en conditions de laboratoire. Agronomie, 1981, 1 (6), pp.449-454. hal-00884279

\author{
HAL Id: hal-00884279 \\ https://hal.science/hal-00884279
}

Submitted on 1 Jan 1981

HAL is a multi-disciplinary open access archive for the deposit and dissemination of scientific research documents, whether they are published or not. The documents may come from teaching and research institutions in France or abroad, or from public or private research centers.
L'archive ouverte pluridisciplinaire HAL, est destinée au dépôt et à la diffusion de documents scientifiques de niveau recherche, publiés ou non, émanant des établissements d'enseignement et de recherche français ou étrangers, des laboratoires publics ou privés. 


\title{
Perturbations dans la reproduction de la pyrale du maïs, Ostrinia nubilalis $\mathrm{Hb}$. (Lépidoptère Pyra- lidae), induites par la présence de la phéromone sexuelle de synthèse en conditions de laboratoire.
}

\author{
Jacques Pierre STOCKEL \\ avec la collaboration technique de Jean-Claude MEYMERIT \\ I.N.R.A. Station de Zoologie, Centre de Recherches de Bordeaux, F33140 Pont de la Maye.
}

\section{RÉSUMÉ}

Pyrale du maïs,

Ostrinia nubilalis,

Confusion sexuelle,

Conditions climatiques,

Ponte,

Taux d'accouplement,

Phéromone synthétique

Diffusion de phéromune,

Fertilité.
Des mâles et des femelles vierges d'Ostrinia nubilalis $\mathrm{Hb}$. sont encagés en présence de diffuseurs de caoutchouc imprégnés par la phéromone sexuelle synthétique : mélange $97: 3$ des isomères $Z$ (cis) et $E$ (trans) de l'acétoxy-1 tétradécène-11, afin d'étudier son rôle sur le comportement reproducteur de cette espèce. Une diffusion moyenne de $350 \mathrm{ng} / \mathrm{h}$ dans $20 \mathrm{~m}^{3}$ entraîne une nette diminution du pouvoir reproducteur des mâles (taux et fréquence des accouplements) et une certaine réduction du dépôt des oufs fertiles par les femelles inséminées. L'utilisation pratique de ces résultats dans l'optique de la lutte par confusion sexuelle contre cet insecte est discutée.

\section{SUMMARY \\ European corn borer, Ostrinia nubilalis, Mating disruption, Climatic conditions, Oviposition, Mating frequency, Synthetic pheromone, Pheromone release, Fertility rate.}

Disturbance in reproduction of european corn borer, Ostrinia nubilalis $\mathrm{Hb}$. (Lepidoptera pyralidae), by pheromone atmosphere in laboratory conditions

\footnotetext{
Virgin males and females of Ostrinia nubilalis $\mathrm{Hb}$. are incaged with rubber septa dispensers impregnated with the synthetic sex pheromone Z:E-97:311 tetradecenyl acetate in view to study its influence on the reproductive behaviour of this insect.

The mean release of $350 \mathrm{ng}$ per hour in a $20 \mathrm{~m}^{3}$ room involves a clear decrease of reproductive ability of males (rate and frequency of mating) and a non negligible decrease of fertile eggs laying by inseminated females. Practical use of these results in view of mating disruption method to control this pest is discussed.
}

\section{INTRODUCTION}

Après l'identification par KLUN \& BRINDLEY (1970) puis KLUN \& RoBINSON (1970) de l'acétoxy-1 tétradécène-11 Z (Z-11 TDA) comme attractif sexuel des mâles de la pyrale du maïs (Ostrinia nubilalis $\mathrm{Hb}$.), les premiers essais de lutte par confusion sexuelle contre cet insecte en conditions naturelles ont été réalisés avec succès par KLUN et al. (1975) à l'aide de l'isomère géométriquement opposé de la phéromone E-11 TDA.

Grâce à l'obtention d'une quantité relativement importante de Z-11 TDA (synthèse réalisée par le Laboratoire des médiateurs chimiques de l'I.N.R.A.), nous avons pu effectuer, en 1976 et 1977, des expérimentations sur l'utilisation de cette méthode dans les conditions de la nature (STOCKEL \& ANGLADE, 1977 ; STOCKEL \& SuREAU, 1977).
Les résultats se sont avérés encourageants. Nous avons ainsi obtenu :

- d'une part la désorientation totale des mâles sauvages au cours de leur quête sexuelle,

- d'autre part une diminution sensible des potentialités sexuelles (nombre d'accouplements et (ou) efficacité des inséminations) peur des mâles d'élevage encagés dans le même environnement phéromonal.

La conséquence agronomique observée fut la diminution d'environ 60 p. 100 de la population sous cages (pontes et chenilles) et une diminution de la population naturelle d'autant plus élevée que la supreficie de la zone traitée était plus grande.

Il paraissait intéressant de compléter de telles études en conditions naturelles par une expérimentation, en condi- 
tions contrôlées du laboratoire, sur des insectes d'élevage afin de comprendre le mécanisme d'action de doses élevées de phéromone sur le comportement reproducteur de cet insecte.

\section{MATÉRIEL ET MÉTHODE}

Les insectes qui sont fournis par l'élevage sur milieu synthétique au laboratoire (MEYMERIT, 1981) proviennent d'une souche indigène récoltée en 1977, à Preignac (Gironde). Chaque année, l'élevage est régénéré par l'apport d'individus nouveaux capturés dans la même loca- lité. Ces pyrales correspondent à la race géographique dont les mâles répondent à la composition isomérique $\mathrm{Z} / \mathrm{E}=97: 3$ de l'acétoxy-1 tétradécène-11, comme nous avons pu le préciser dans un essai antérieur de piégeage sexuel en conditions naturelles (STOCKEL \& MEYMERIT, 1978).

L'expérimentation a lieu en chambre climatisée, en conditions constantes de température $\left(20^{\circ} \mathrm{C}\right)$ et d'humidité relative $(80-90$ p. 100$)$, pour une photopériode de $16 \mathrm{~h}$ de jour. Les insectes vierges âgés de 24 à 48 h sont groupés dans des enceintes grillagées de taille et modèles différents (tabl. 1) munies de pondoirs (lames de papier sulfurisé) disposés verticalement, soit en présence soit en absence de diffuseurs de phéromone dans les chambres climatisées.

TABLEAU 1

Conditions expérimentales de chacun des essais

Experimental conditions in each of the 2 tests

\begin{tabular}{|c|c|c|c|c|}
\hline Essai & $\begin{array}{l}\text { Groupement } \\
\text { des insectes }\end{array}$ & Type de cage & $\begin{array}{l}\text { Quantité } \\
\text { de phéromone }\end{array}$ & $\begin{array}{l}\text { Volume sous } \\
\text { ambiance phéromonale }\end{array}$ \\
\hline I & $\begin{array}{l}2 \text { mâles } \\
1 \text { femelle }\end{array}$ & Cagette $7 \times 7 \times 9 \mathrm{~cm}$ & $3,6 \mathrm{~g}$ & $20 \mathrm{~m}^{3}$ \\
\hline II & $\begin{array}{l}33 \text { mâles } \\
33 \text { femelles }\end{array}$ & Cage $30 \times 40 \times 30 \mathrm{~cm}$ & $3,6 \mathrm{~g}$ & $20 \mathrm{~m}^{3}$ \\
\hline
\end{tabular}

La phéromone utilisée, mélange isomérique du 11 TDA dans la proportion $\mathrm{Z} / \mathrm{E}=97: 3$, est répartie dans 36 diffuseurs de caoutchouc (bouchons de tube à allergie LEUNE) chargés unitairement de $100 \mathrm{mg}$. Ces diffuseurs sont pesés, avant chargement puis chaque semaine, af in de déterminer la diffusion de phéromone par la mesure de la perte pondérale.

Deux essais ont été réalisés successivement dans des conditions expérimentales légèrement différentes décrites ci-dessous :

\section{Essai $n^{\circ} 1$ :}

Les insectes sont groupés par trios ( 2 mâles et 1 femelle) dans des cagettes grillagées $(7 \times 7 \times 9 \mathrm{~cm})$ disposées sur des plateaux dans deux chambres climatisées séparées (traitée-témoin). L'ambiance phéromonale est assurée par la répartition des 36 diffuseurs entre et autour des cagettes (fig. 1).

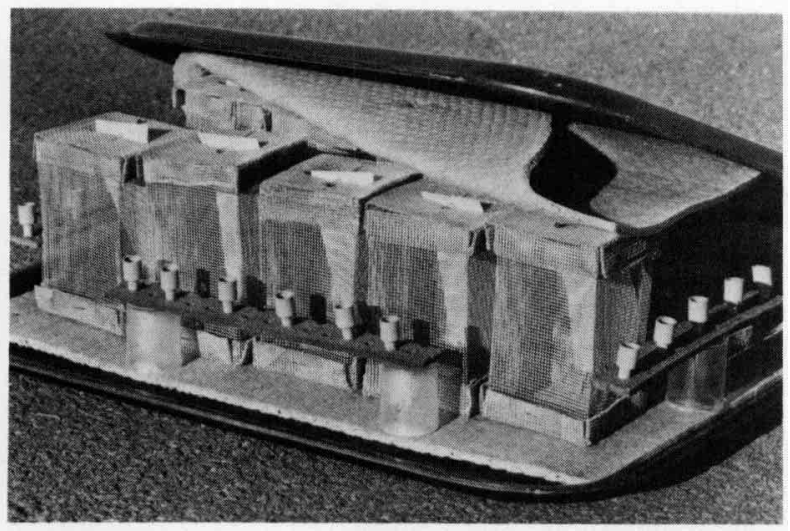

Figure 1

Dispositif expérimental d'élevage utilisé pour l'essai $n^{\prime \prime} 1$.

Experimental apparatus used for the first bioessay.

\section{Essai $n^{\circ} 2$ :}

Les insectes sont groupés par 33 couples dans des cages de pontes utilisées pour l'élevage de masse de l'insecte au laboratoire $(30 \times 40 \times 30 \mathrm{~cm})$ et disposées comme précédemment dans les 2 chambres climatisées séparées (fig. 2).

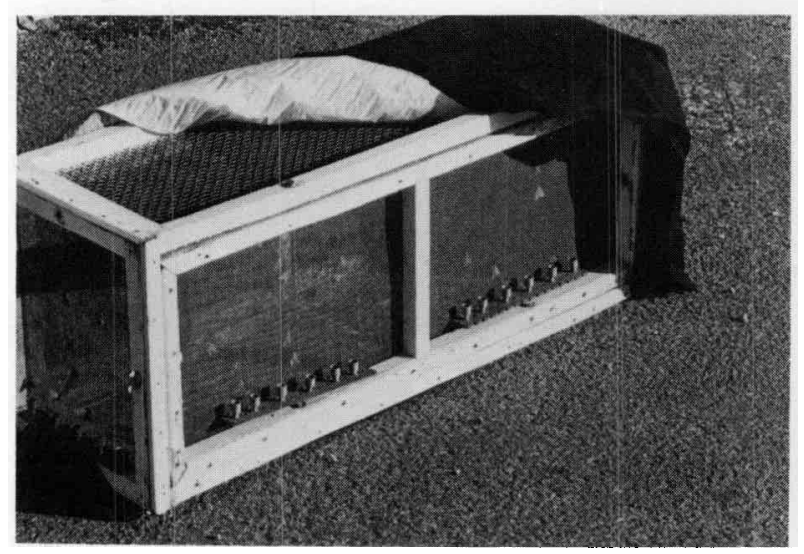

Figure 2

Dispositif expérimental d'élevage utilisé pour l'essai $n^{\circ} 2$.

Experimental apparatus used for the second bioessay.

Dans tous les cas, les œufs furent dénombrés tous les 2 et $3 \mathrm{j}$. Les pontes étaient alors placées à $25^{\circ} \mathrm{C}$ et 90 p. 100 H.R. afin de préciser, 7 à $8 \mathrm{j}$ plus tard, le taux de fertilité déterminé par l'apparition du stade "tête noire » dans les œufs.

En outre, la mortalité des insectes était notée chaque jour et les femelles mortes étaient disséquées en vue d'apprécier le taux d'insémination par le nombre de spermatophores présents dans leur bourse copulatrice. 


\section{RESULTATS}

\section{A) Diffusion de phéromone}

La mesure de la perte pondérale représentée graphiquement (fig. 3) indique que la diffusion est très comparable pendant toute la durée de l'expérience dans les 2 essais. La diffusion moyenne est respectivement de :

- $8,073 \mathrm{mg} / \mathrm{j}$ soit $336 \mathrm{ng} / \mathrm{h}$ pour l'essai $\mathrm{n}^{\circ} 1$,

$-9,031 \mathrm{mg} / \mathrm{j}$ soit $376 \mathrm{ng} / \mathrm{h}$ pour l'essai $\mathrm{n}^{\circ} 2$

ce qui correspond à une diffusion moyenne d'environ $10 \mathrm{ng} / \mathrm{h}$ par diffuseur pour chaque essai.

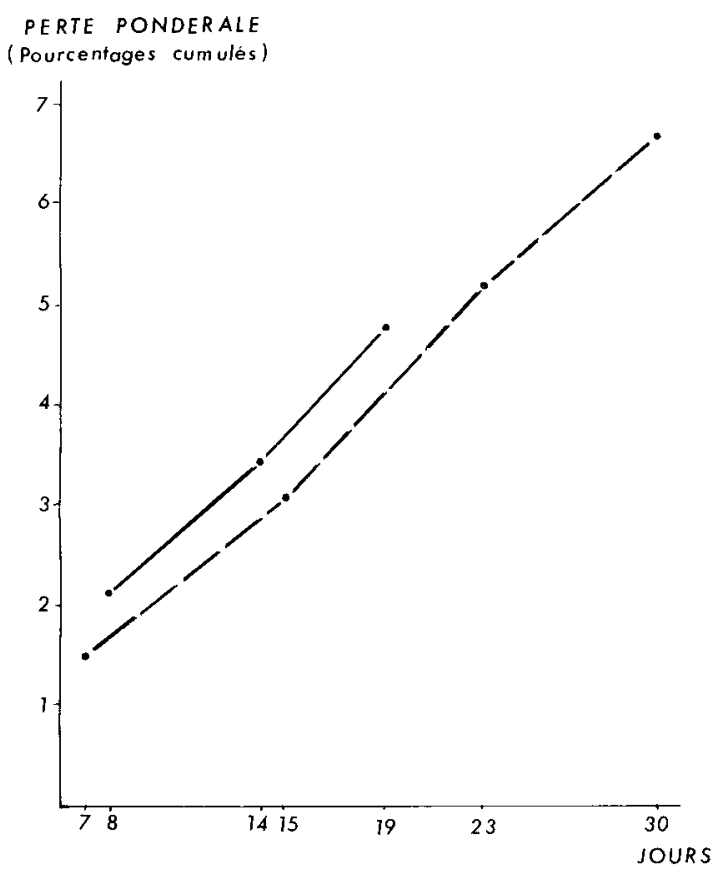

Figure 3

Diffusion de la phéromone évaluée par la perte pondérale des

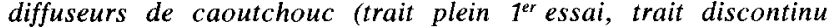
$2^{2}$ essai).

Diffusion of pheromone calculated by weight loss of rubber releasers (continuous line : 1st bioessay, discontinuous line : 2nd bioessay).

\section{B) Pouvoir reproducteur du mâle d' $O$. nubilalis}

\section{a) Taux d'accouplement}

Pour l'ensemble des 2 essais, il y eut 100 femelles «témoins» contre 99 "traitées" (phéromone). Les résultats des dissections: nombre de femelles inséminées et, chez ces dernières, nombre de spermatophores trouvés dans leur bourse copulatrice sont représentés dans le tableau 2.

Dans chacun des 2 essais, quel que soit le lot considéré, il apparaît un pourcentage de femelles non inséminées. Comme nous remarquerons que ce pourcentage de non-accouplement est toujours plus élevé chez les femelles "traitées" (R2) que chez les femelles "témoins » (R1), il nous semble possible d'en attribuer la différence (R2-R1) pour chaque essai à l'effet de l'ambiance phéromonale sur le pouvoir copulateur des mâles.

D'une manière globale, la réduction d'accouplement est, dans ces conditions, voisine de 65 p. 100

\section{b) Fréquence des accouplements}

Le pouvoir reproducteur des mâles peut aussi s'exprimer par la fréquence des accouplements chez les femelles inséminées (tabl. 3).

\section{TABLEAU 3}

Influence de la présence de phéromone sur la fréquence des inséminations chez les femelles de pyrale du maïs

Influence of pheromone atmosphere on mating frequency

\begin{tabular}{|c|c|c|c|}
\hline \multirow{2}{*}{ Essai } & \multicolumn{3}{|c|}{ Nombre de spermatophores par femelle inséminée } \\
\hline & Témoin & Traité & Différence U-Test \\
\hline I & 1,42 & 1,00 & Seuil 0,05 \\
\hline II & 1,19 & 1,00 & N.S. \\
\hline Moyenne & 1,27 & 1,00 & 0,05 \\
\hline
\end{tabular}

Quel que soit l'essai, on peut constater que si la présence de phéromone diminue sensiblement la proportion des femelles inséminées, elle empêche aussi tout accouplement multiple chez ces dernières. En revanche, il n'est pas rare

TABLEAU 2

Influence de la présence de phéromone ( $Z / E=97: 3$ 11-TDA) sur la réduction du taux d'accouplement Influence of pheromone atmosphere $(Z / E=97: 311$-TDA) on the reduction of mating rate

\begin{tabular}{|c|c|c|c|c|c|c|c|c|c|c|c|}
\hline \multirow{3}{*}{ Essai } & \multicolumn{5}{|c|}{ Témoin } & \multicolumn{5}{|c|}{ Traité (Z11 TDA) } & \multirow{3}{*}{$\begin{array}{c}\text { Réduction } \\
\text { d'accouplement } \\
\text { R2-R1 }\end{array}$} \\
\hline & \multirow{2}{*}{ Total } & \multirow{2}{*}{$\begin{array}{l}q \text { insé- } \\
\text { minées }\end{array}$} & \multirow{2}{*}{$\begin{array}{l}\text { Total } \\
\text { Spm }\end{array}$} & \multicolumn{2}{|c|}{ q non inséminées } & \multirow{2}{*}{ Total } & \multirow{2}{*}{$\begin{array}{l}\text { q insé- } \\
\text { minées }\end{array}$} & \multirow{2}{*}{$\begin{array}{c}\text { Total } \\
\text { Spm }\end{array}$} & \multicolumn{2}{|c|}{ @ non inséminées } & \\
\hline & & & & Total & $\%=\mathrm{R} 1$ & & & & Total & $\%=\mathrm{R} 2$ & \\
\hline I & 34 & 33 & 47 & 1 & 2,9 & 33 & 14 & 14 & 19 & 57,6 & $54,7 \%$ \\
\hline II & 66 & 59 & 70 & 7 & 10,6 & 66 & 13 & 13 & 53 & 80,3 & $69,7 \%$ \\
\hline $\begin{array}{l}\text { Total } \\
\% \text { moyen }\end{array}$ & 100 & 92 & 117 & 8 & 8,0 & 99 & 27 & 27 & 72 & 72,7 & $64,7 \%$ \\
\hline
\end{tabular}


de rencontrer des femelles inséminées 2 et même 3 fois chez les femelles témoins (fig. 4).

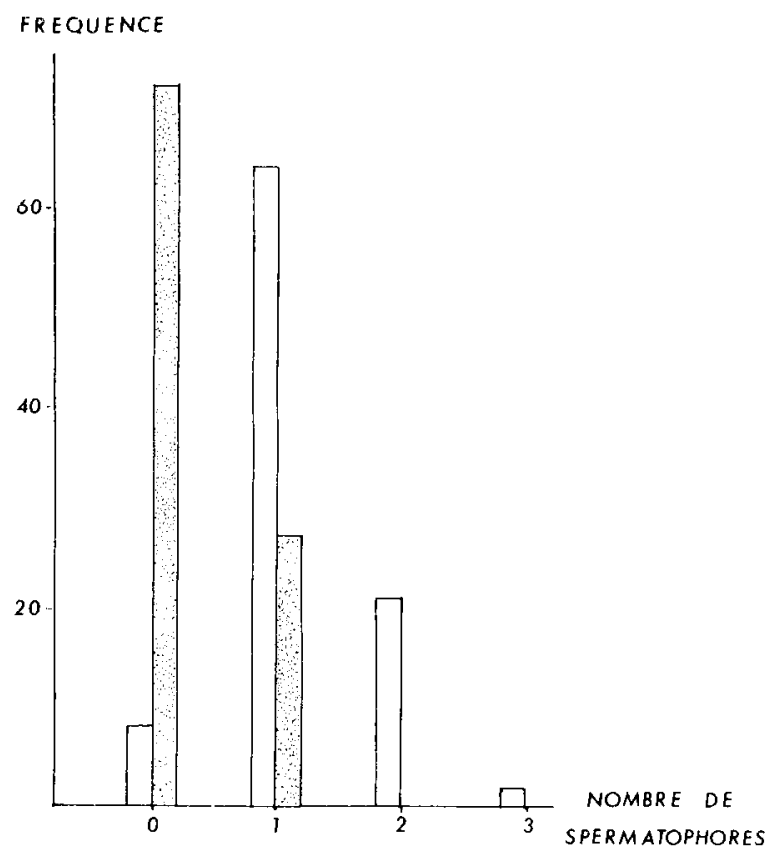

Figure 4

Distribution de toutes les femelles selon la fréquence des accouplements (gris : phéromone, blanc : témoin).

Distribution of all females according to mating frequency (grey: pheromone, white : control).

\section{C) La fécondité}

a) La fécondité moyenne par femelle (tabl. 4) paraît fortement affectée, de manière globale pour l'ensemble des 2 essais, par la présence de phéromone. La moyenne de ponte qui est de 125,6 œufs par femelle est abaissée à 38,3 en ambiance phéromonale, soit à 33 p. 100 environ. L'analyse réalisée sur les pontes des seules femelles individualisées (essai $\left.n^{\prime \prime} 1\right)$ confirme l'importance de cette différence (P-0,001). Il faut remarquer que cette différence provient à la fois du plus grand nombre de femelles non inséminées (ne pondant pas ou seulement des oufs stériles) et aussi d'un plus grand nombre de femelles faiblement pondeuses et d'un faible effectif de femelles fortement pondeuses (fig. 5).

\section{b) La fécondité par femelle selon son état sexuel}

Compte tenu des observations signalées plus haut, une étude plus fine semblait nécessaire. Elle fut réalisée sur les pontes des femelles individualisées du $1^{\mathrm{er}}$ essai.

Quel que soit le lot "témoin " ou «traité ", les résultats (tabl. 5) montrent qu'il existe seulement 3 catégories de femelles inséminées pondeuses :

1) Femelles pondant seulement des cufs fertiles;

2) Femelles pondant seulement des cufs stériles;

3) Femelles pondant des cufs fertiles et des oufs stériles.

A cela il faut ajouter que certaines femelles vierges peuvent déposer un nombre d'œufs stériles non négligeable.

TABLEAU 4

Influence globale de l'ambiance pheromenale sur la feconditc do. nubilalis General influence of pheromone atmosphere on oriposition of (1). nuthlialis

\begin{tabular}{|c|c|c|c|c|c|}
\hline \multicolumn{2}{|c|}{ Essai } & \multirow{2}{*}{$\begin{array}{c}\begin{array}{c}\text { Nombre de } \\
\text { femelles }\end{array} \\
34 \\
33\end{array}$} & \multirow{2}{*}{$\begin{array}{c}\begin{array}{c}\text { Total œufs } \\
\text { pondus }\end{array} \\
\begin{array}{l}7485 \\
2185\end{array}\end{array}$} & \multirow{2}{*}{$\begin{array}{c}\begin{array}{c}\text { Ponte moyenne } \\
\text { par femelle }\end{array} \\
220,1 \\
66,2\end{array}$} & \multirow{2}{*}{$\begin{array}{c}\begin{array}{c}\text { Signification } \\
\text { (U-Test) }\end{array} \\
0,001\end{array}$} \\
\hline I & $\begin{array}{l}\text { Témoin } \\
\text { Traité }\end{array}$ & & & & \\
\hline II & $\begin{array}{l}\text { Témoin } \\
\text { Traité }\end{array}$ & $\begin{array}{l}66 \\
66\end{array}$ & $\begin{array}{l}5073 \\
1158\end{array}$ & $\begin{array}{l}76,9 \\
17,5\end{array}$ & - \\
\hline Total & $\begin{array}{l}\text { Témoin } \\
\text { Traité }\end{array}$ & $\begin{array}{r}100 \\
99\end{array}$ & $\begin{array}{r}12558 \\
3343\end{array}$ & $\begin{array}{r}125,6 \\
33,8\end{array}$ & - \\
\hline
\end{tabular}

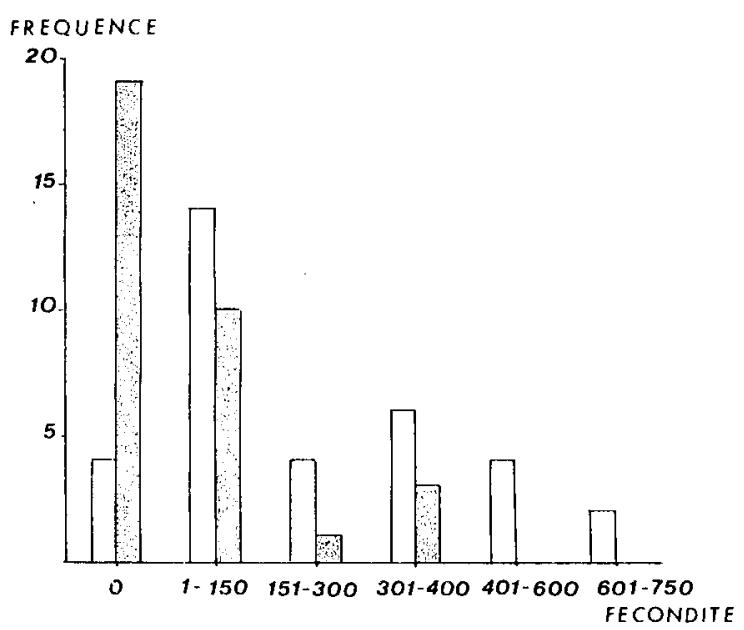

Étant donné la faiblesse des effectifs de femelles réparties dans ces diverses catégories, nous n'avons comparé que les pontes fertiles des femelles de la $1^{\text {re }}$ et de la $3^{\mathrm{e}}$ catégories, en présence et en absence de phéromone.

Les différences pour ces 2 catégories de femelles ne sont significatives qu'aux seuils respectifs de 10 et 20 p. 100 . Elles ne peuvent donc qu'indiquer une tendance commune à un effet réducteur du dépôt d'œufs fertiles par les femelles placées en ambiance phéromonale.

Distribution des femelles de l'essai $n^{o} 1$ selon leur fécondité (gris : phéromone, blanc: témoin).

Distribution of the first data females according to their oviposition rate (grey: pheromone, white: control). 
TABLEAU 5

Influence de l'ambiance phéromonale sur la ponte des femelles du premier essai $(\omega=$ cufs, Fert. $=$ fertile, Stér. $=$ stérile $)$

Influence of pheromone atmosphere on first bioessay female oviposition $(\omega=$ eggs, Fert. $=$ fertile, Ster. $=$ sterile $)$

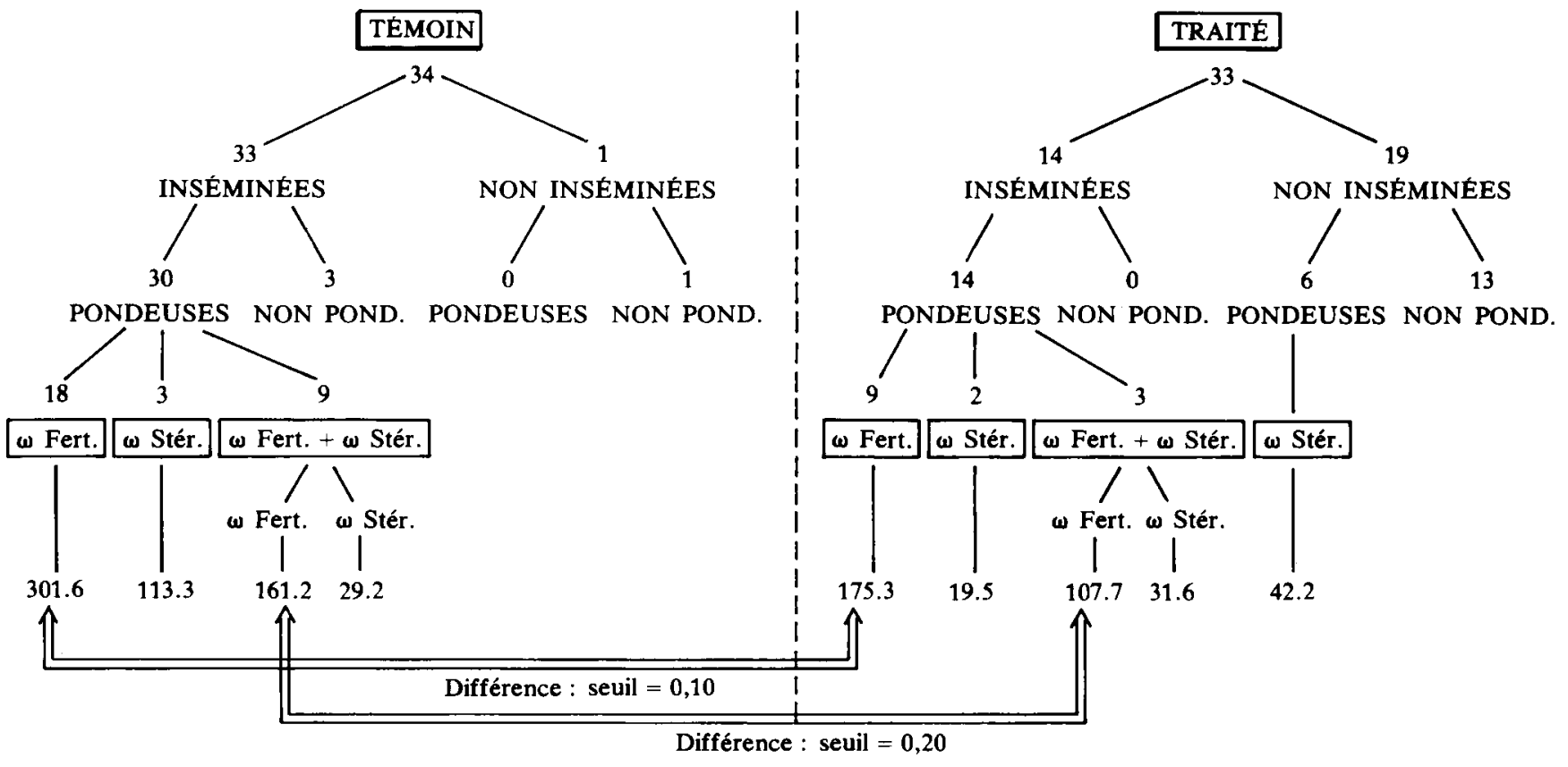

Ainsi, la phéromone agirait aussi sur la physiologie même de la femelle au niveau du processus de la fécondation et (ou) de celui de l'émission d'œufs.

\section{D) La longévité des femelles}

Bien que pour chacun des 2 essais, quel que soit le traitement, les mâles paraissaient mourir généralement avant les femelles, nous n'avons enregistré que la mortalité de ces dernières.

Il est clair (tabl. 6) que, dans le $1^{\text {er }}$ essai, l'ambiance phéromonale a considérablement allongé la durée de vie moyenne des femelles. Deux raisons peuvent en être la cause :

- les femelles vierges d'O. nubilalis vivraient plus longtemps que les femelles inséminées pondeuses et nous venons de voir que l'ambiance phéromonale diminue les taux d'accouplements;

- la présence de phéromone entraînerait, seulement chez certaines femelles, un retard à l'accouplement qui aurait pour effet de prolonger d'autant leur durée de vie.

Dans le $2^{\mathrm{e}}$ essai en revanche, cet allongement n'apparaît plus significatif mais les conditions expérimentales y sont différentes, en particulier par le groupement des insectes par 33 couples.

\section{TABLEAU 6}

Influence de l'ambiance phéromonale sur la longévité des femelles Influence of pheromone atmosphere on longevity of females

\begin{tabular}{clcc}
\hline \hline Essai & $\begin{array}{c}\text { Durée de vie } \\
\text { des femelles (jours) } \\
\text { Témoin }\end{array}$ & $\begin{array}{c}\text { Signification de la } \\
\text { Traitérence (U-Test) }\end{array}$ \\
\hline I & 10,21 & 14,21 & 0,001 \\
II & 10,76 & 10,92 & NS \\
\hline \hline
\end{tabular}

\section{DISCUSSION. CONCLUSION}

Dans les conditions expérimentales décrites, une diffusion moyenne de $350 \mathrm{ng} / \mathrm{h}$ pour $20 \mathrm{~m}^{3}$ de Z-11 TDA entraîne une diminution importante du pouvoir reproducteur des mâles d' $O$. nubilalis. Leur aptitude à copuler diminue d'environ 65 p. 100 , surtout dans le cas d'insectes groupés. Cela se traduit inévitablement par une diminution importante de la fécondité totale des femelles.

Comme on observe par ailleurs une certaine diminution des pontes fertiles chez les femelles inséminées en présence de phéromone, il est vraisemblable que cette dernière n'agit pas seulement sur la physiologie sexuelle du mâle mais aussi sur celle de la femelle.

Cette hypothèse serait confirmée par la tendance à une augmentation générale de la durée de vie des femelles soumises à la phéromone. Ceci peut être dû soit à une augmentation du pourcentage de femelles restant vierges et vivant plus longtemps que les autres, comme nous avons pu le vérifier par ailleurs sur un autre insecte (STOCKEL, 1973), soit à un retard à l'accouplement et, par suite, à la ponte comme l'ont observé ROEHRICH \& CARLES (1977) sur Lobesia botrana Schiff.

Quoi qu'il en soit, c'est la descendance potentielle de l'insecte en ambiance phéromonale qui constitue le résultat pratique de cette étude, c'est-à-dire le nombre d'œufs fertiles déposés par l'ensemble des femelles présentes. Ainsi, en dépit de conditions expérimentales différentes dans chacun des 2 essais, il apparaît que le nombre moyen d'œufs fertiles pondus par femelle diminue sensiblement de 106,5 à 22,3 en ambiance de phéromone. Cela se traduit alors par une réduction du nombre moyen d'œufs fertiles par femelle de l'ordre de 80 p. 100 (tabl. 7).

Ces résultats de laboratoire confirment et permettent d'expliquer ceux que nous avions obtenus en conditions naturelles sous cages (STOCKEL \& SUREAU, 1977).

Nous n'avons pas rapporté dans cette note les résultats dans le même sens d'un essai précédent portant sur une 
TABLEAU 7

Influence générale de l'ambiance phéromonale sur la descendancé potentielle do. nubilalis General influence of pheromone atmosphere on potential progeny of 0 . nubilalis

\begin{tabular}{|c|c|c|c|c|c|}
\hline \multicolumn{2}{|c|}{ Essai } & $\begin{array}{l}\text { Nombre total } \\
\text { œufs fertiles }\end{array}$ & $\begin{array}{l}\text { Nombre total } \\
\text { de femelles }\end{array}$ & $\begin{array}{l}\text { Nombre moyen d'œufs } \\
\text { fertiles/femelle }\end{array}$ & $\begin{array}{l}\% \text { de réduction du } \\
\text { nombre } \omega \text { fertiles } / q\end{array}$ \\
\hline I & $\begin{array}{l}\text { Témoin } \\
\text { Traité }\end{array}$ & $\begin{array}{l}6881 \\
1862\end{array}$ & $\begin{array}{l}34 \\
33\end{array}$ & $\begin{array}{r}202,4 \\
56,4\end{array}$ & 72,2 \\
\hline II & $\begin{array}{l}\text { Témoin } \\
\text { Traité }\end{array}$ & $\begin{array}{r}3766 \\
342\end{array}$ & $\begin{array}{l}66 \\
66\end{array}$ & $\begin{array}{r}57,1 \\
5,2\end{array}$ & 90,9 \\
\hline Total & $\begin{array}{l}\text { Témoin } \\
\text { Traité }\end{array}$ & $\begin{array}{r}10647 \\
2204\end{array}$ & $\begin{array}{r}100 \\
99\end{array}$ & $\begin{array}{r}106,5 \\
22,3\end{array}$ & 79,1 \\
\hline
\end{tabular}

centaine de femelles, car les conditions expérimentales dues au confinement (double enceinte) furent dans l'ensemble défavorables à la ponte, masquant ainsi en partie l'effet dû à la phéromone.

Il sera nécessaire toutefois de poursuivre cette étude de laboratoire afin de préciser la relation « dose de phéromoneréduction des accouplements ». Une telle étude pourra être conduite en diffusiomètre, appareil conçu et réalisé pour des essais biologiques de ce type par CARLES et al. (1979).

Pour aussi incomplets qu'ils soient, ces résultats de laboratoire confirment nos observations antérieures dans les conditions de la nature. En rejoignant ceux de ROEHRICH \& CARLES (1977) sur Lobesia botrana, ils permettent de mieux cerner les limites de l'application pratique de la confusion sexuelle comme méthode de lutte contre les insectes. Il est clair, en effet, que si la rencontre des sexes se produit tout de même en ambiance de phéromone, les autres séquences de la reproduction peuvent se dérouler presque normalement assurant ainsi la survie de l'espèce. Une telle méthode de lutte est donc limitée et il semble qu'elle ne pourra jamais être utilisée seule mais en complément d'une technique de lutte classique.

Reçu le 12 janvier 1981. Accepté le 20 février 1981.

\section{REMERCIEMENTS}

Nous remercions M. C. Descoins du Laboratoire des médiateurs chimiques de l'I.N.R.A. pour nous avoir procuré la phéromone ainsi que MM. P. Anglade et R. ROEHRICH pour leurs conseils dans la discussion des résultats et la rédaction de cette note, enfin J. P. Carles pour les photographies.

\section{REFERENCES BIBLIOGRAPHIQUES}

Carles J. P., Fleurat Lessard F., Roehrich R., 1979. Un appareil pour les essais biologiques de comportement de Lépidoptères exposés à des doses élevées d'attractif sexuel. Biol. Comport., 4, 205-217.

Klun J. A., Brindley T. A., 1970. Cis-11-tetradecenyl acetate, a sex stimulant of the European Corn Borer. J. econ. Entomol., 63, 779-780.

Klun J. A., Robinson J. F., 1970. Inhibition of the European Corn Borer mating by cis-11-tetradecenyl acetate, a borer sex stimulant. J. econ. Entomol. 63, 1281-1283.

Klun J. A., Chapman O. L., Mattes K. C., Beroza M., 1975. European Corn Borer and Redbanded Leafroller : Disruption of reproduction behaviour. Environ. Entomol., 4, 871-876.

Meymerit J. C., 1981. Technique d'élevage de la pyrale du maïs, Ostrinia nubilalis Hb. (Lep. Pyralidae), sur milieu artificiel pour l'obtention programmée d'adultes vierges. Cahiers de liaison de l'O.P.I.E. (sous presse).

Roehrich R., Carles J. P., 1977. Perturbations dans la reproduction de Lobesia botrana Schiff. (Lepidoptera Tortricidae) induites par la présence de la phéromone sexuelle de synthèse. C.R. Acad. Sci., Paris (D), 285, 237-239.

Stockel J., 1973. Influence des relations sexuelles et du milieu trophique de l'adulte sur la reproduction de Sitotroga cerealella Oliv. (Lép. Gelechiidae) ; conséquences écologiques. Thèse Docteur-Ingénieur $\mathrm{n}^{\circ} 3$, Univ. de Tours, $175 \mathrm{p}$.

Stockel J., Anglade P., 1977. Influence de la concentration en isomères cis et trans dans la phéromone sexuelle d'Ostrinia nubilalis Hb. sur le comportement d'orientation de cette espèce et sur celui de Pyrausta aurata Sc. C.R. Acad. Sci., Paris (D), 85, 61-64.

Stockel J., Meymerit J. C., 1978. Premières observations de l'étude éco-éthologique de la pyrale du maïs en vue de l'utilisation de la méthode de confusion sexuelle contre cet insecte en conditions naturelles. In "Les phéromones sexuelles des insectes et les médiateurs chimiques". Antibes, nov. 1978, Publ. I.N.R.A., 61-63.

Stockel J., Sureau F., 1977. Seconde année d'essais de lutte par confusion sexuelle contre Ostrinia nubilalis Hb. (Lép. Pyralidae) dans les conditions naturelles. In «Les phéromones sexuelles des Insectes». Avignon, oct. 1977, Publ. I.N.R.A., 151-157. 